Research Article

\title{
On the Metric Dimension of Generalized Tensor Product of Interval with Paths and Cycles
}

\author{
Song Li, ${ }^{1}$ Jia-Bao Liu $\mathbb{D}^{2},{ }^{2}$ and Mobeen Munir ${ }^{3}{ }^{3}$ \\ ${ }^{1}$ School of Computer Science and Technology, Hefei Normal University, Anhui, Hefei 230601, China \\ ${ }^{2}$ School of Mathematics and Physics, Anhui Jianzhu University, Hefei 230601, China \\ ${ }^{3}$ Department of Mathematics, Division of Science and Technology, University of Education, Lahore, Pakistan \\ Correspondence should be addressed to Mobeen Munir; mmunir@ue.edu.pk
}

Received 24 January 2020; Revised 22 June 2020; Accepted 4 July 2020; Published 30 August 2020

Academic Editor: Mehdi Ghatee

Copyright (c) 2020 Song Li et al. This is an open access article distributed under the Creative Commons Attribution License, which permits unrestricted use, distribution, and reproduction in any medium, provided the original work is properly cited.

The concept of minimum resolving set for a connected graph has played a vital role in Robotic navigation, networking, and in computer sciences. In this article, we investigate the values of $m$ and $n$ for which $\left(P_{2} \otimes^{m}\right) P_{n}$ and $\left(P_{2} \otimes^{m}\right) C_{n}$ are connected and find metric dimension in this case. We also conclude that, for each $m$, we obtain a new regular family of constant metric dimension. We also give a basis for these graphs and presentation of resolving vector in general closed form with respect to the basis.

\section{Introduction}

The concept of metric dimension and resolving sets were initially drafted for the metric spaces in 1953 in [1], p. 95 but did not receive much attention because of the linear continuum nature of standard Euclidean spaces $\mathbb{R}^{n}$. Later, Slater in $[2,3]$ and Harary and Melter in [4] used these ideas of uniquely determining the location of an intruder in a network and graphs. This idea also paved the way for searching uniquely the receiver of a message on a network. Since then the resolving sets have been widely investigated, see, for instance, $[5,6]$. The resolving sets also have useful applications in diverse areas including network discovery and verification [7], strategies for the mastermind games [8], and applications to problems of pattern recognition, image processing, and digital geometry [5]. Chartrand et al. discussed resolvability and its connection with metric dimension of graphs in $[9,10]$. Similarly, on another node, a real-world problem is the study of networks which is not centrally controlled but rather distributed. So, it becomes relatively difficult and costly to obtain a map of all nodes and the links between them. One possible solution is to view network from various local positions and combine them to obtain a good approximation for the real network, [11]. Metric dimension has some applications in this respect as well. Buczkowski et al. discussed a general idea of $k$-dimensional graphs [12]. First, we introduce general terminology related to a graph.

A graph $G$ is an ordered pair $(V, E)$, where $V$ is the vertex set and $E$ is the edge. The distance between vertices $v, w \in V$, denoted by $d(v, w)$, is defined as the length of the shortest path between $v$ and $w$, and the diameter of $G$, denoted by $\operatorname{dia}(G)$, is defined as the maximum distance among all pairs of vertices in $G$. A vertex $x \in V$ resolves a pair of vertices $v, w \in V$ if $d(v, x) \neq d(w, x)$. A set of vertices $W \subseteq V$ resolves $G$ if each pair of distinct vertices of $G$ is resolved by some vertex in $W$. The set $W$ is called the resolving set of $G$ if it resolves $G$. A resolving set $W$ of $G$ with the least cardinality is called metric basis of $G$, and this cardinality is the called metric dimension of $G$ denoted by $\beta(G)$. It is a common fact now that $\beta$ is unique although we may have many metric bases. Actually the choice of a particular matric basis leads us towards the unique coordinate representation of all vertices of a graph. For an ordered subset $W=\left\{w_{1}, w_{2}, \ldots, w_{n}\right\}$ of vertices and a vertex $v$ in a connected graph $G$, the representation of $v$ with respect to $W$ is the ordered $k$-tuple.

A lot of work has already been carried out on computation of metric dimension and its other variants related ideas of graphs. Buczkowski et al. [12] calculated the dimension of wheel $W_{n}$ and arrived at the result that $\operatorname{dim}\left(W_{n}=((2 n+\right.$ 2)/5)) for $n \geq 7$. Caceres et al. [13] computed the dimension of 
fan graphs, $f_{n}$, and proved that, for $n \geq 7, \operatorname{dim}\left(f_{n}\right)=$ $((2 n+2) / 5)$. Tomescu and Javaid proved that the dimension of Jahangir graphs $J_{2 n}$ to be $\operatorname{dim}\left(J_{2 n}\right)=(2 n / 3)$ for $n \geq 4$ [14]. Imran et al. computed the dimension of circulant graphs in [15]. Hussain et al. computed bounds for the metric and partition dimension of generalized Mobius ladders in [16].

In the abovementioned cases, metric dimension of all graphs depends upon the number of vertices in the graph. In contrast, one is interested in the list of all families of graphs in which metric dimension remains constant which seems to be an important feature of the family. Authors in [17, 18] discussed some families of graphs with constant metric dimension. Another aspect is the metric dimension of a graph that is some kind of product of two other graphs. One is often interested in relating the dimension of the product graphs with dimensions of the component graphs. Cartesian product of two graphs $G$ and $H$, denoted by $G \square H$ is a graph such that the vertex set of $G \square H$ is the Cartesian product $V(G) \times V(H)$ as a set, and any two vertices $\left(u, u^{\prime}\right)$ and $\left(v, v^{\prime}\right)$ are adjacent in $G \square H$ if and only if either $u=v$ and $u^{\prime}$ is adjacent with $v^{\prime}$ in $H$ or $u^{\prime}=v^{\prime}$ and $u$ is adjacent with $v$ in $G$. The metric dimension of the Cartesian products of graphs has been studied in [19]. Authors proved that the metric dimension of $G \square G$ is tied in a strong sense to the minimum order of a so-called doubly resolving set in $G$. Authors also computed bounds on $G \square H$ for many examples of $G$ and $H$. Authors in [20] recursively defined generalized corona product and derived some important results about this product in terms of the dimension of components involved, whereas, in [21], authors computed strong metric dimension of this corona product. Another product is the tensor product, $G \otimes H$ of graphs $G$ and $H$ which is a graph such that the vertex set of $G \otimes H$ is the Cartesian product $V(G) \otimes V(H)$; any two vertices $\left(u, u^{\prime}\right)$ and $\left(v, v^{\prime}\right)$ are adjacent in $G \otimes H$ if and only if $u^{\prime}$ is adjacent with $v^{\prime}$ and $u$ is adjacent with $v$. For example, the tensor product of $P_{2}$ with

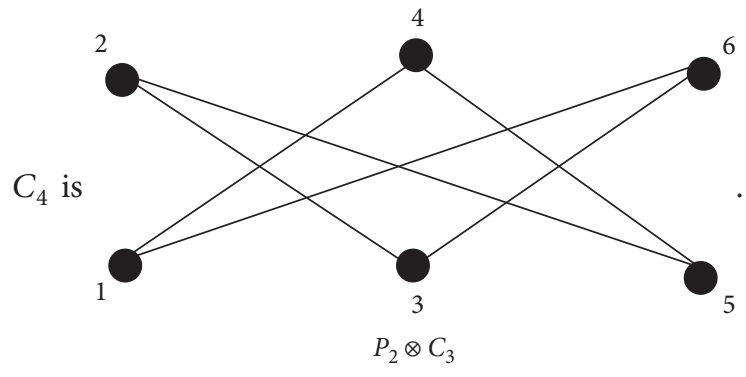

Moradi discussed the tensor product of some graphs in [22]. Jannesari and Omoomi computed metric dimension of lexicographic product of some graphs by introducing a new parameter known as adjacency dimension, [23]. Saputro et al. further extended these results for other graphs in [24]. Javaid et al. computed metric dimension of co-normal product of graphs in [25].

In this paper, we discuss $n$-copies of tensor product of $P_{2}$ with $P_{n}$ and $C_{n}$. This type of product is relatively less studied in the literature as compared to the Cartesian product. In the first stage, we decide about the connectedness of this product. At the second step, we compute the metric dimension of the connected graphs among them. We like to remark that metric dimension of the tensor product of two graphs has not been extensively studied over the years.

\section{Main Results}

We focus first on $P_{2} \otimes{ }^{m} P_{n}$. We compute minimal resolving set for it allowing $\infty$ as distance between two vertices when there does not exist any path connecting them so they are in different components of a graph. The interesting question is the value of $m$ for which the graph $\left(P_{2} \otimes^{m}\right) P_{n}$ is connected. The answer, however, is no in this case. Existence of $\infty$ as any component of resolving vector clearly indicates that the graph is disconnected. Although we cannot talk about the metric dimensions in these cases but cardinality of minimum removability does make sense as it shows the connectedness of a graph. We actually give the closed representation of all vertices with respect to our selected metric basis in each case.

\section{Theorem 1}

(i) $\forall m$ and $n \geq 2,\left(P_{2} \otimes^{m}\right) P_{n}$ is disconnected

(ii) Cardinality of the minimal resolving set is $2^{m}$

Proof. Let $W=\left\{\left(u_{i}, v_{j, 1}, v_{j, 2}, \ldots, v_{j, m}\right)\right\}$, where $\left(u_{i}\right)$ are vertices $P_{n}$ and $v_{j, k}$ is the vertex of $P_{2}$ of $K$ th component. We show that $W$ is a resolving set. For this, we give the representation of any vertex of $V\left(P_{2} \otimes{ }^{m} P_{n}\right)$ with respect to $W$ :

$$
\begin{aligned}
& r\left(\left.\left(w_{2 j+1}, u_{i, 1}, u_{i, 2}, \ldots, u_{m-1}, v_{1}\right)\right|_{W}\right)= \begin{cases}\left(a_{x, x}=j-1,\right. & 0 \leq j \leq n-2,1 \leq i \leq 2,1 \leq x \leq 2^{m-1}, \\
\left.a_{x, y}=\infty\right), & 1 \leq i \leq 2,1 \leq x \leq 2^{m-1}, 1 \leq y \leq 2^{m},\end{cases} \\
& r\left(\left.\left(w_{2 j+1}, u_{i, 1}, u_{i, 2}, \ldots, u_{m-1}, v_{2}\right)\right|_{W}\right)= \begin{cases}\left(a_{x, x}=j-1,\right. & 0 \leq j \leq n-2,1 \leq i \leq 2,1+2^{m-1} \leq x \leq 2^{m}, \\
\left.a_{x, y}=\infty\right), & 1 \leq i \leq 2,1+2^{m-1} \leq x \leq 2^{m}, 1 \leq y \leq 2^{m},\end{cases} \\
& r\left(\left.\left(w_{2 j}, u_{i, 1}, u_{i, 2}, \ldots, u_{m-1}, v_{1}\right)\right|_{W}\right)= \begin{cases}\left(a_{x, x-1+2^{m}}=j-1,\right. & 1 \leq j \leq n-1,1 \leq i \leq 2,1+2^{m-1} \leq x \leq 2^{m}, \\
\left.a_{x, y}=\infty\right), & 1 \leq i \leq 2,1 \leq y \leq 2^{m}, 1+2^{m-1} \leq x \leq 2^{m},\end{cases}
\end{aligned}
$$


Now, we consider the minimality of the resolving set. If we delete $\left\{\left(u_{1,1}, u_{1,2}, \ldots, u_{1, m}, v_{1}\right)\right\}$ from $W$, then $r\left(\left(w_{1}, u_{1,1}, \ldots\right.\right.$, $\left.\left.u_{1, m-1}, v_{1}\right)\left.\right|_{W}\right)=r\left(\left.\left(w_{3}, u_{1,1}, \ldots, u_{1, m-1}, v_{1}\right)\right|_{W}\right)=(\infty, \infty, \ldots$, $\infty, \infty)$. So, cardinality of minimum recoverability $\left(\left(P_{2} \otimes^{m}\right)\right.$ $\left.P_{n}\right)$ is $2^{m}$.

Example 1. If $m=2$ and $n=3$, then $\beta\left(P_{2} \otimes P_{2} \otimes P_{3}\right)=4$ and $W=\left\{\left(w_{1}, u_{1}, v_{1}\right),\left(w_{1}, u_{2}, v_{1}\right),\left(w_{2}, u_{1}, v_{1}\right),\left(w_{2}, u_{1}, v_{1}\right)\right\}$

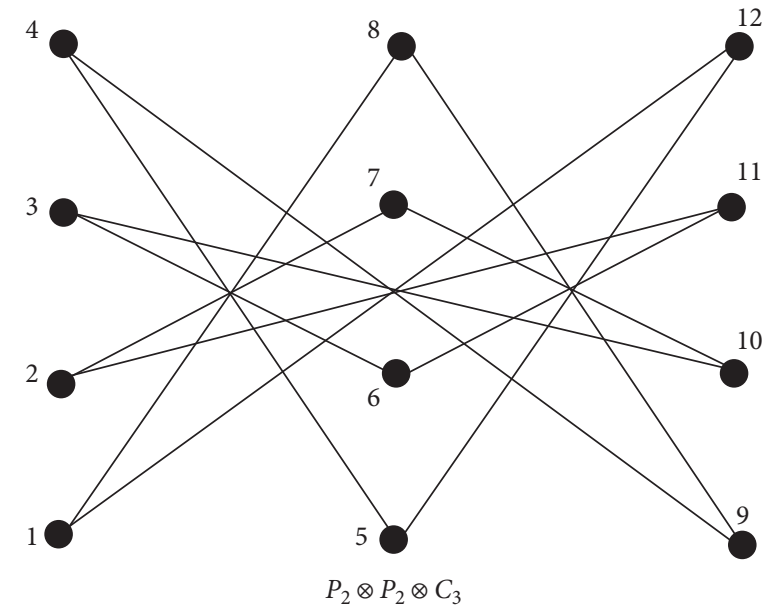

resolving vectors with respect to $W$ are

$$
\begin{aligned}
& \left(w_{1}, u_{1}, v_{1}\right)=(0, \infty, 2, \infty), \\
& \left(w_{1}, u_{1}, v_{2}\right)=(\infty, 0, \infty, 2), \\
& \left(w_{1}, u_{2}, v_{1}\right)=(\infty, 3, \infty, 1), \\
& \left(w_{1}, u_{2}, v_{2}\right)=(3, \infty, 1, \infty), \\
& \left(w_{2}, u_{1}, v_{1}\right)=(2, \infty, 0, \infty), \\
& \left(w_{2}, u_{1}, v_{2}\right)=(\infty, 2, \infty, 0), \\
& \left(w_{2}, u_{2}, v_{1}\right)=(\infty, 1, \infty, 3), \\
& \left(w_{2}, u_{2}, v_{2}\right)=(1, \infty, 3, \infty), \\
& \left(w_{3}, u_{1}, v_{1}\right)=(2, \infty, 2, \infty), \\
& \left(w_{3}, u_{1}, v_{2}\right)=(\infty, 2, \infty, 2), \\
& \left(w_{2}, u_{1}, v_{1}\right)=(\infty, 1, \infty, 1), \\
& \left(w_{3}, u_{2}, v_{2}\right)=(1, \infty, 1, \infty) .
\end{aligned}
$$

Now, we turn our attention to the $P_{2} \otimes^{m} C_{n}$. In contrast to the above result, we have an interesting result here. For

and $m=1$ and $n \equiv 1(\bmod 2)$, our graph is connected. Moreover, we also give metric dimension in this case as well.

\section{Theorem 2}

(1) $\beta\left(P_{2} \otimes C_{n}\right)=2$.

(2) Cardinality of the minimal resolving set:

$$
\beta\left(\left(P_{2} \otimes{ }^{m} C_{n}\right)\right)= \begin{cases}2^{m}, & \text { if } n \text { is odd, } \\ 2^{m+1}, & \text { if } n \text { is even. }\end{cases}
$$

(3) $P_{2} \otimes{ }^{m} C_{n}$ is connected iff $m=1$ and $n \equiv 1(\bmod 2)$.

Proof. (1) Let $W=\left\{\left(u_{1}, v_{1}\right),\left(u_{2}, v_{1}\right)\right\}$ be set of vertices, where $\left(v_{k}\right)$ are vertices of $P_{2}$ and $\left(u_{1}\right)$ and $\left(u_{2}\right)$ are adjacent vertices of $C_{n}$. We show that $W$ is a resolving set. For this, we give the representation of any vertex of $\left.V\left(P_{2} \otimes C_{n}\right)\right|_{W}$ with respect to $W$ :

$$
\begin{aligned}
r\left(\left.\left(u_{2 i+1}, v_{1}\right)\right|_{W}\right) & =(2 i,|2 i-1|), \quad 0 \leq i \leq n-2, \\
r\left(\left.\left(u_{2 i}, v_{1}\right)\right|_{W}\right) & =(n-2 i+1, n-2 i+2), \quad 1 \leq i \leq n-2, \\
r\left(\left.\left(u_{2 i+1}, v_{2}\right)\right|_{W}\right) & =(n-2 i, n-2 i+1), \quad 0 \leq i \leq n-2, \\
r\left(\left.\left(u_{2 i}, v_{2}\right)\right|_{W}\right) & =(2 i-1,2 i-2), \quad 1 \leq i \leq n-2 .
\end{aligned}
$$

We see that all the representations are distinct, implying that $\operatorname{dim}\left(P_{2} \otimes C_{n}\right)=2$. Now, we prove that $W$ is a minimal resolving set. If we take $W=\left\{\left(u_{1}, v_{1}\right)\right\}$, we receive two similar resolving vectors: $r\left(\left.\left(u_{2}, v_{1}\right)\right|_{W}\right)=r\left(\left(u_{2 k+1}\right.\right.$, $\left.\left.v_{1}\right)\left.\right|_{W}\right)=2 k$, which indicates dimension is not 1 . So, $\beta\left(P_{2} \otimes C_{n}\right)$ is 2 .

(2) Case 1 (when $n$ is odd): we compute the general form of resolving vector in this case.

Let $W=\left\{\left(u_{i}, v_{j, 1}, v_{j, 2}, \ldots, v_{j, m}\right)\right\}$, where $v_{k}$ are vertices of $P_{2}$ and $\left(u_{t}\right)$ and $\left(u_{2}\right)$ are vertices of $C_{n}$. We show that $W$ is a resolving set. For this, we give the representation of any vertex of $V\left(P_{2} \otimes{ }^{m} C_{2 k+1}\right)$ with respect to $W$ :

$$
\begin{aligned}
r\left(\left.\left(w_{2 l+1}, u_{i, 1}, \ldots, u_{i, m-1}, v_{1}\right)\right|_{W}\right) & = \begin{cases}\left(a_{x, x}=2 l,\right. & 0 \leq l \leq k, \\
a_{x, x+2^{m-1}}=2 k+1-|2 l-1|, & 0 \leq l \leq k, \\
\left.a_{x, y}=\infty\right),\end{cases} \\
r\left(\left.\left(w_{2 l}, u_{i, 1}, \ldots, u_{i, m-1}, v_{1}\right)\right|_{W}\right) & = \begin{cases}\left(a_{x, x}=2 k-2 l+3,\right. & 1 \leq l \leq 2 k-2, \\
a_{x, x+2^{m-1}}=2 l+1, & 1 \leq l \leq 2 k-2, \\
\left.a_{x, y}=\infty\right),\end{cases} \\
r\left(\left.\left(w_{2 l+1}, u_{i, 1}, \ldots, u_{i, m-1}, v_{2}\right)\right|_{W}\right) & = \begin{cases}\left(a_{x, 1+2^{m}-x}=2 l-1,\right. & 0 \leq l \leq k, \\
a_{x, 3+2^{m}-x}=2 k-2 l+2, & 0 \leq l \leq k, \\
\left.a_{x, y}=\infty\right), & 1 \leq l \leq k,\end{cases} \\
r\left(\left.\left(w_{2 l}, u_{i, 1}, \ldots, u_{i, m-1}, v_{2}\right)\right|_{W}\right) & = \begin{cases}\left(a_{x, 1+2^{m}-x}=2 l-1,\right. & 1 \leq l \leq k, \\
a_{x, 3+2^{m}-x}=2 k-2 l+3, & \\
\left.a_{x, y}=\infty\right),\end{cases}
\end{aligned}
$$


where $i=1,2,1 \leq x \leq 2^{m-1}$, and $1 \leq y \leq 2^{m}$.

We see that all the representations are distinct, implying that $\beta\left(P_{2} \otimes_{m} C_{n}\right) \leq 2^{m}, m \geqq 2$. Now, we prove that $W$ is a minimal resolving set. If we delete $\left\{\left(u_{i}, v_{j, 1}, v_{j, 2}, \ldots, v_{j, m}\right)\right\}$ from $W$, we receive two similar resolving vectors: $r\left(\left(w_{1}, u_{1,1}\right.\right.$, $\left.\left.\ldots, u_{1, m-1}, v_{1}\right)\left.\right|_{W}\right)=r\left(\left.\left(w_{3}, u_{1,1}, \ldots, u_{1, m-1}, v_{1}\right)\right|_{W}\right)=(\infty, \ldots$, $\infty, 2 k, \infty, \ldots, \infty)$, where $2 k$ appears at $2^{m-1}$ th position, which shows $W$ is the minimal resolving vector.

Example 2. If $m=2$ and $n=3$, then $\beta\left(P_{2} \otimes P_{2} \otimes C_{3}\right)=4$ and $W=\left\{\left(w_{1}, u_{1}, v_{1}\right),\left(w_{1}, u_{2}, v_{1}\right),\left(w_{2}, u_{1}, v_{1}\right),\left(w_{2}, u_{2}, v_{1}\right)\right\} . P_{2} \otimes$ $P_{2} \otimes C_{3}$ and resolving vectors with respect to $W$ are

$$
\begin{aligned}
& \left(w_{1}, u_{1}, v_{1}\right)=(0, \infty, \infty, \infty), \\
& \left(w_{1}, u_{1}, v_{2}\right)=(\infty, \infty, \infty, 1), \\
& \left(w_{1}, u_{1}, v_{3}\right)=(2, \infty, \infty, \infty), \\
& \left(w_{1}, u_{2}, v_{1}\right)=(\infty, 0, \infty, \infty), \\
& \left(w_{1}, u_{2}, v_{2}\right)=(\infty, \infty, 1, \infty), \\
& \left(w_{1}, u_{2}, v_{3}\right)=(\infty, 2, \infty, \infty),
\end{aligned}
$$

$$
\begin{aligned}
& \left(w_{2}, u_{1}, v_{1}\right)=(\infty, \infty, 0, \infty), \\
& \left(w_{2}, u_{1}, v_{2}\right)=(\infty, 1, \infty, \infty), \\
& \left(w_{2}, u_{1}, v_{3}\right)=(\infty, \infty, 2, \infty), \\
& \left(w_{2}, u_{2}, v_{1}\right)=(\infty, \infty, \infty, 2), \\
& \left(w_{2}, u_{2}, v_{2}\right)=(1, \infty, \infty, \infty), \\
& \left(w_{2}, u_{2}, v_{3}\right)=(\infty, \infty, \infty, 0) .
\end{aligned}
$$

Remark 1. The above example and picture show that graph is disconnected. This holds generally for this case.

Case 2 (when $n$ is even): let $W=\left\{\left(u_{i}, v_{j, 1}, v_{j, 2}, \ldots, v_{j, m}\right)\right\}$. We show that $W$ is a resolving set. For this, we give the representation of any vertex of $V\left(P_{2} \otimes{ }^{m} C_{2 k}\right)$ with respect to $W$.

When $1 \leq i, j \leq 2,1 \leq x \leq 2^{m}$, and $1 \leq y \leq 2^{m-1}$,

$$
r\left(\left.\left(w_{2 l+1}, u_{i, 1}, \ldots, u_{i, m-1}, v_{1}\right)\right|_{W}\right)=\left\{\begin{array}{l}
\left(a_{x, x}= \begin{cases}2 l, & 0 \leq l \leq 1, \\
l, & l=2, k=3, \\
l+2, & l=2, k>3, \\
2 k-2 l, & 3 \leq l \leq 2 k-1, k \geq 4,\end{cases} \right. \\
a_{x, x+2^{m+1}-1}= \begin{cases}|2 l-1|, & 0 \leq l \leq 1, \\
2 k-2 l+1, & 2 \leq l \leq k-1, k \geq 3, \\
\left.a_{x, y}=\infty\right) .\end{cases}
\end{array}\right.
$$

When $2^{m} \leq x \leq 2^{m+1}$,

$$
r\left(\left.\left(w_{2 l+1}, u_{i, 1}, \ldots, u_{i, m-1}, v_{2}\right)\right|_{W}\right)=\left\{\begin{array}{l}
\left(a_{x, x-1}= \begin{cases}2 l, & 0 \leq l \leq 1, \\
l, & l=2, k=3, \\
l+2, & l=2, k>3, \\
2 k-2 l, & 3 \leq l \leq 2 k-1, k \geq 4,\end{cases} \right. \\
a_{x, x+1}= \begin{cases}|2 l-1|, & 0 \leq l \leq 1, \\
2 k-2 l+1, & 2 \leq l \leq k-1, k \geq 3 .\end{cases}
\end{array}\right.
$$


When $1 \leq i, j \leq 2,1 \leq x \leq 2^{m}$, and $1 \leq y \leq 2^{m-1}$,

$$
r\left(\left.\left(w_{2 l}, u_{i, 1}, \ldots, u_{i, m-1}, v_{1}\right)\right|_{W}\right)=\left\{\begin{array}{l}
\left(a_{x, x}= \begin{cases}2 l-1, & l=1, l=2, k>2, \\
2 l-3, & l=2, k=2, \\
2 k-2 l+1, & 3 \leq l \leq 2 k, k \geq 3,\end{cases} \right. \\
a_{x, x+1}= \begin{cases}2 l-2, & 1 \leq l \leq 2, k \geq 2, \\
2 k+2 l+2, & 3 \leq l \leq k, k \geq 3,\end{cases} \\
\left.a_{x, y}=\infty\right) .
\end{array}\right.
$$

When $2^{m} \leq x \leq 2^{m+1}$,

$$
r\left(\left.\left(w_{2 l}, u_{i, 1}, \ldots, u_{i, m-1}, v_{1}\right)\right|_{W}\right)=\left\{\begin{array}{l}
\left(a_{x, x}= \begin{cases}2 l-1, & l=1, l=2, k>2, \\
2 l-3, & l=2, k=2, \\
2 k-2 l+1, & 3 \leq l \leq 2 k, k \geq 3,\end{cases} \right. \\
a_{x, x+1}= \begin{cases}2 l-2, & 1 \leq l \leq 2 k, k \geq 2, \\
2 k+2 l+2, & 3 \leq l \leq k, k \geq 3,\end{cases} \\
\left.a_{x, y}=\infty\right) .
\end{array}\right.
$$

If we delete $\left\{\left(u_{i}, v_{j, 1}, v_{j, 2}, \ldots, v_{j, m}\right)\right\}$ from $W$, we receive two similar resolving vectors $r\left(\left.\left(w_{1}, u_{1,1}, \ldots, u_{1, m-1}, v_{1}\right)\right|_{W}\right)=$ $r\left(\left.\left(w_{3}, u_{1,1}, \ldots, u_{1, m-1}, v_{1}\right)\right|_{W}\right)=(\infty, \ldots, \infty, 2 k, \infty, \ldots, \infty)$, where $2 k$ appears at $2^{m-1}$ th position, which shows $W$ is the minimal resolving set.

(3) From the above resolving pattern, we can argue this result by presence of $\infty$ at any place.

Remark 2. The same result can be proved by [22] by the agreement that the tensor product of two graphs is disconnected when both factors are bipartite and connected.

Example 3. If $m=2$ and $n=4$, then $\beta\left(P_{2} \otimes P_{2} \otimes P_{4}\right)=8$ and $W=\left\{\left(w_{1}, u_{1}, v_{1}\right),\left(w_{1}, u_{1}, v_{2}\right),\left(w_{1}, u_{2}, v_{1}\right),\left(w_{1}, u_{2}, v_{2}\right),\left(w_{2}, u_{1}\right.\right.$, $\left.\left.v_{1}\right),\left(w_{2}, u_{1}, v_{2}\right),\left(w_{2}, u_{2}, v_{1}\right),\left(w_{2}, u_{2}, v_{2}\right)\right\}$ and resolving vectors with respect to $W$ are

$$
\begin{aligned}
& \left(w_{1}, u_{1}, v_{1}\right)=(0, \infty, \infty, \infty, \infty, \infty, \infty, 1), \\
& \left(w_{1}, u_{1}, v_{2}\right)=(\infty, 0, \infty, \infty, \infty, \infty, 1, \infty), \\
& \left(w_{1}, u_{2}, v_{1}\right)=(\infty, \infty, 0, \infty, \infty, 1, \infty, \infty), \\
& \left(w_{1}, u_{2}, v_{2}\right)=(\infty, \infty, \infty, 0,1, \infty, \infty, \infty), \\
& \left(w_{2}, u_{1}, v_{1}\right)=(\infty, \infty, \infty, 1,0, \infty, \infty, \infty), \\
& \left(w_{2}, u_{1}, v_{2}\right)=(\infty, \infty, 1, \infty, \infty, 0, \infty, \infty), \\
& \left(w_{2}, u_{2}, v_{1}\right)=(\infty, 1, \infty, \infty, \infty, \infty, 0, \infty), \\
& \left(w_{2}, u_{2}, v_{2}\right)=(1, \infty, \infty, \infty, \infty, \infty, \infty, 0), \\
& \left(w_{3}, u_{1}, v_{1}\right)=(2, \infty, \infty, \infty, \infty, \infty, \infty, 1),
\end{aligned}
$$

$$
\begin{aligned}
& \left(w_{3}, u_{1}, v_{2}\right)=(\infty, 2, \infty, \infty, \infty, \infty, 1, \infty), \\
& \left(w_{3}, u_{2}, v_{1}\right)=(\infty, \infty, 2, \infty, \infty, 1, \infty, \infty), \\
& \left(w_{3}, u_{2}, v_{2}\right)=(\infty, \infty, \infty, 2,1, \infty, \infty, \infty), \\
& \left(w_{4}, u_{1}, v_{1}\right)=(\infty, \infty, \infty, 1,2, \infty, \infty, \infty), \\
& \left(w_{4}, u_{1}, v_{2}\right)=(\infty, \infty, 1, \infty, \infty, 2, \infty, \infty), \\
& \left(w_{4}, u_{2}, v_{1}\right)=(\infty, 1, \infty, \infty, \infty, \infty, 2, \infty), \\
& \left(w_{4}, u_{2}, v_{2}\right)=(1, \infty, \infty, \infty, \infty, \infty, \infty, 2)
\end{aligned}
$$

\section{Conclusions}

In the end, we like to summarize our main outcomes. First, noticeable point is the disconnectedness of all $P_{2} \otimes{ }^{m} P_{n}$. The sum and substance of this article is the connectedness of $P_{2} \otimes{ }^{m} C_{n}$ for $m=1$ and $n \equiv 1(\bmod 2)$ and metric dimension in this case is 2 . So, we obtain a regular family of graph with constant metric dimension 2, which is decomposable into paths and cycles. From network point of view, only this family is significant. However, for the remaining families, we can find minimum resolving set and its cardinality. We conclude this article with

$$
\beta\left(\left(P_{2} \otimes{ }^{m} C_{n}\right)\right)= \begin{cases}2^{m}, & \text { if } n \text { is odd } \\ 2^{m+1}, & \text { if } n \text { is even. }\end{cases}
$$




\section{Data Availability}

No data were used to support the findings of the study.

\section{Conflicts of Interest}

The authors declare that they have no conflicts of interest.

\section{Authors' Contributions}

All authors contributed equally to the writing of this paper. All authors read and approved the final manuscript.

\section{Acknowledgments}

This study was supported by Key Project of Anhui University Natural Fund: Study on Image Semantic Extraction and Optimization Model Based on Voronoi Graph and Random Graph (KJ2013A327) and Wisdom Classroom Project in the University-Level Quality Engineering of Hefei Normal University: Application of Modern Educational Information Technology (2018zhkt10).

\section{References}

[1] L. M. Blumenthal, Theory and Applications of Distance Geometry, Chelsea Publishing Company, New York, NY, USA, 1953.

[2] P. J. Slater, "Leaves of trees," Congressus Numerantium, vol. 14, pp. 549-559, 1975.

[3] P. J. Slater, "Dominating and refrences sets in graphs," Journal of Mathematical and Physical Sciences, vol. 22, pp. 445-455, 1998.

[4] F. Harary and R. A. Melter, "On the metric dimension of a graph,” ARS Combinatoria, vol. 2, pp. 191-195, 1976.

[5] R. A. Melter and I. Tomescu, "Metric bases in digital geometry," Computer Vision, Graphics, and Image Processing, vol. 25, no. 1, pp. 113-121, 1984.

[6] G. Chartrand, D. Erwin, G. L. Johns, and P. Zhang, "Boundary vertices in graphs," Discrete Mathematics, vol. 263, no. 1-3, pp. 25-34, 2003.

[7] S. Khuller, B. Raghavachari, and A. Rosenfeld, "Landmarks in graphs," Discrete Applied Mathematics, vol. 70, no. 3, pp. 217-229, 1996.

[8] A. Bogomolny and D. Greenwell, "Cut the knot: invitation to mastermind," 1999, http://www.maa.org/editorial/knot/ mastermind.html.

[9] G. Chartrand, L. Eroh, M. A. Johnson, and R. Oellermann, "Resolvability in graphs and the metric dimension of a graph," Discrete Applied Mathematics, vol. 105, no. 1-3, pp. 99-113, 2000.

[10] G. Chartrand, C. Poisson, and P. Zhang, "Resolvability and the upper dimension of graphs," Computers \& Mathematics with Applications, vol. 39, no. 12, pp. 19-28, 2000.

[11] P. Frank and R. Silverman, "Remarks on detection problems," The American Mathematical Monthly, vol. 74, no. 2, pp. 171-173, 1967.

[12] P. S. Buczkowski, G. Chartrand, C. Poisson, and P. Zhang, "On $k$-dimensional graphs and their bases," Periodica Mathematica Hungarica, vol. 46, no. 1, pp. 9-15, 2003.

[13] J. Caceres, C. Hernando, M. Mora et al., "On the metric dimension of some families of graphs," Electronic Notes in Discrete Mathematics, vol. 22, pp. 129-133, 2005.
[14] I. Tomescu and I. Javaid, "On the metric dimension of the Jahangir graph," Bulletin Mathematique de la Societe des Sciences Mathematiques de Roumanie, vol. 50, no. 98, pp. 371-376, 2007.

[15] M. Imran, A. Q. Baig, S. A. U. H. Bokhary, and I. Javaid, "On the metric dimension of circulant graphs," Applied Mathematics Letters, vol. 25, no. 3, pp. 320-325, 2012.

[16] Z. Hussain, J. A. Khan, M. Munir, M. S. Saleem, and Z. Iqbal, "Sharp bounds for partition dimension of generalized Möbius ladders," Open Mathematics, vol. 16, pp. 1283-1290, 2018.

[17] I. Javaid, M. T. Rahim, and K. Ali, "Families of regular graphs with constant metric dimension," Utilitas Mathematica, vol. 75, pp. 21-33, 2008.

[18] M. Imran, A. Q. Baig, and A. Ahmed, "Families of plane graphs with constant metric dimension," Utilitas Mathematica, vol. 88, pp. 43-57, 2012.

[19] J. Caceres, C. Hernando, M. Mora et al., "On the metric dimension of Cartesian product of graphs," SIAM Journal on Discrete Mathematics, vol. 2, no. 21, pp. 423-441, 2007.

[20] G. Yero, D. Kuziak, and J. A. Rodryguez Velazquez, "On the metric dimension of corona product graphs," Computers and Mathematics with Applications, vol. 61, no. 9, pp. 2793-2798, 2011.

[21] D. Kuziak, I. G. Yero, and J. A. Rodrguez-Velazquez, "On the strong metric dimension of corona product graphs and join graphs," Discrete Applied Mathematics, vol. 161, no. 7-8, pp. 1022-1027, 2013.

[22] S. Moradi, "A note on tensor product of graphs," Iranian Journal of Mathematical Sciences and Informatics, vol. 7, no. 1, pp. 73-81, 2012.

[23] M. Jannesari and B. Omoomi, "The metric dimension of the lexicographic product of graphs," Discrete Mathematics, vol. 312, no. 22, pp. 3349-3356, 2012.

[24] S. W. Saputro, R. Simanjuntak, S. Uttunggadewa et al., "The metric dimension of the lexicographic product of graphs," Discrete Mathematics, vol. 313, pp. 1045-1051, 2013.

[25] I. Javaid, S. U. Rehman, and M. Imran, "Bounds on the domination number and the metric dimension of co-normal product of graphs," Journal of Inequalities and Applications, vol. 2018, no. 1, p. 162, 2018. 